\title{
SUPERSOLVABLE REFLECTION ARRANGEMENTS
}

\author{
TORSTEN HOGE AND GERHARD RÖHRLE
}

(Communicated by Lev Borisov)

\begin{abstract}
Let $\mathcal{A}=(\mathcal{A}, V)$ be a complex hyperplane arrangement and let $L(\mathcal{A})$ denote its intersection lattice. The arrangement $\mathcal{A}$ is called supersolvable, provided its lattice $L(\mathcal{A})$ is supersolvable, a notion due to Stanley. Jambu and Terao showed that every supersolvable arrangement is inductively free, a notion previously due to Terao. So this is a natural subclass of this particular class of free arrangements.

Suppose that $W$ is a finite, unitary reflection group acting on the complex vector space $V$. Let $\mathcal{A}=(\mathcal{A}(W), V)$ be the associated hyperplane arrangement of $W$. In a forthcoming paper by the authors, we determine all inductively free reflection arrangements.

The aim of this paper is to classify all supersolvable reflection arrangements. Moreover, we characterize the irreducible arrangements in this class by the presence of modular elements of rank 2 in their intersection lattice.
\end{abstract}

\section{INTRODUCTION}

Let $\mathcal{A}=(\mathcal{A}, V)$ be a complex hyperplane arrangement and let $L(\mathcal{A})$ denote its intersection lattice. We say that $\mathcal{A}$ is supersolvable, provided $L(\mathcal{A})$ is supersolvable; see Definition 2.2. Jambu and Terao [JT84, Thm. 4.2] have shown that every supersolvable arrangement is inductively free, a notion due to Terao [Ter80; see Definition 2.1.

Now suppose that $W$ is a finite, unitary reflection group acting on the complex vector space $V$. Let $\mathcal{A}=(\mathcal{A}(W), V)$ be the associated hyperplane arrangement of $W$. Terao [Ter80] has shown that each reflection arrangement $\mathcal{A}$ is free and that the multiset of exponents $\exp \mathcal{A}$ of $\mathcal{A}$ is given by the coexponents of $W$; cf. OT92, §6]. There is the stronger notion of inductive freeness referred to above; cf. Definition 2.1. In HR12, we classified all inductively free reflection arrangements; cf. Theorem 1.1. In this paper we determine the subclass of all supersolvable reflection arrangements: apart from the braid and rank 2 arrangements, these consist only of the reflection arrangements of the groups $G(r, p, \ell)$ for $r, \ell \geq 2$ and $p \neq r$; cf. Theorem 1.2. In addition, the irreducible arrangements of this nature are characterized by the presence of a modular rank 2 element in the intersection lattice; see Theorem 1.3 .

Within the theory of hyperplane arrangements supersolvability is a rather strong condition, as it implies essentially every desirable property, such as factored, free,

Received by the editors September 14, 2012 and, in revised form, January 7, 2013.

2010 Mathematics Subject Classification. Primary 20F55, 52C35, 14N20; Secondary 13N15.

Key words and phrases. Complex reflection groups, reflection arrangements, free arrangements, supersolvable arrangements. 
fiber-type, $K(\pi, 1)$, rational $K(\pi, 1)$, etc.; see [FR85, (2.8)] for details. We briefly discuss three relevant properties.

Firstly, the Poincaré polynomial $\pi(\mathcal{A}, t)$ of the lattice $L(\mathcal{A})$ of a supersolvable arrangement $\mathcal{A}$ factors into linear terms over $\mathbb{Z}[t]$, thanks to a theorem of Stanley Sta72; see also OT92, Thm. 2.63]. Precisely, $\pi(\mathcal{A}, t)$ factors into linear terms as follows:

$$
\pi(\mathcal{A}, t)=\prod_{i=1}^{\ell}\left(1+b_{i} t\right),
$$

where the coefficients $b_{i}$ are positive integers.

Secondly, as mentioned above, a supersolvable arrangement $\mathcal{A}$ is always inductively free [JT84, Thm. 4.2]; see also OT92, Thm. 4.85]. So we can talk about the exponents $\exp \mathcal{A}$ of $\mathcal{A}$ in this case; cf. \$2.2. In the factorization of $\pi(\mathcal{A}, t)$ above, the occurring coefficients $b_{i}$ are precisely the exponents of $\mathcal{A}$ [T92, Thm. 4.137].

Thirdly, if $\mathcal{A}$ is supersolvable, then $\mathcal{A}$ is a $K(\pi, 1)$-arrangement, for Falk and Randell [FR85] proved that fiber-type arrangements are always $K(\pi, 1)$ (cf. OT92, Prop. 5.12]), and by work of Terao [Ter86, the classes of fiber-type arrangements and of supersolvable arrangements coincide (cf. OT92, Thm. 5.112]). Therefore, every supersolvable arrangement is $K(\pi, 1)$.

Since a supersolvable arrangement is inductively free, we only need to consider the latter for our classification. We recall the main result from HR12.

Theorem 1.1. For $W$ a finite complex reflection group, the reflection arrangement $\mathcal{A}(W)$ of $W$ is inductively free if and only if $W$ does not admit an irreducible factor isomorphic to a monomial group $G(r, r, \ell)$ for $r, \ell \geq 3, G_{24}, G_{27}, G_{29}, G_{31}, G_{33}$, or $G_{34}$.

Jambu and Terao have already observed that $\mathcal{A}\left(D_{4}\right)$ is not supersolvable JT84, Ex. 5.5]. Thus, supersolvable reflection arrangements form a proper subclass of the class of inductively free reflection arrangements.

It is straightforward to see that any rank 2 arrangement is supersolvable; cf. Remark 2.3. In 1962, Fadell and Neuwirth FN62 showed that the braid arrangement is fiber-type, and in 1973, Brieskorn [Br73] proved this for the reflection arrangement of the hyperoctahedral group. In 1983, using Brieskorn's iterated fibration method, Orlik and Solomon [OS83, §4] observed that indeed every $\mathcal{A}(G(r, p, \ell))$ is fiber-type, for $r, \ell \geq 2$ and $p \neq r$. So $\mathcal{A}$ is supersolvable in each of these cases. Our classification asserts that this list is actually complete.

Theorem 1.2. For $W$ a finite complex reflection group, $\mathcal{A}(W)$ is supersolvable if and only if any irreducible factor of $W$ is of rank at most 2 or is isomorphic either to a Coxeter group of type $A_{\ell}$ or $B_{\ell}$ for $\ell \geq 3$ or to a monomial group $G(r, p, \ell)$ for $r, \ell \geq 3$ and $p \neq r$.

In 1962, Fadell and Neuwirth FN62 proved that the braid arrangement $\mathcal{A}\left(A_{\ell}\right)$ is $K(\pi, 1)$. In 1973, Brieskorn Br73 extended this result to a large class of Coxeter groups and conjectured that this is the case for every Coxeter group. This was proved subsequently by Deligne [Del72, who showed that every simplicial arrangement is $K(\pi, 1)$. All reflection arrangements $\mathcal{A}(W)$ have been known to be $K(\pi, 1)$ since the late 1980s, with the exception of the ones for the six exceptional groups listed in Theorem [1.1 see [0T92, §6.6]. Since these arrangements are not 
inductively free, they are not supersolvable (or fiber-type), and so one cannot easily deduce that they are also $K(\pi, 1)$. These outstanding cases were settled only recently by Bessis $\mathrm{Be} 06]$.

The definition of supersolvability of $\mathcal{A}$ entails the existence of modular elements in $L(\mathcal{A})$ of any possible rank; see $\$ 2.4$ for the notion of modular elements. Strikingly, our second main result shows that irreducible, supersolvable reflection arrangements are characterized merely by the presence of a modular element of rank 2 .

Theorem 1.3. For $W$ a finite, irreducible complex reflection group of rank at least $2, \mathcal{A}(W)$ is supersolvable if and only if there exists a modular element of rank 2 in its lattice $L(\mathcal{A}(W))$.

The condition of irreducibility in Theorem 1.3 is necessary; see Remark 2.6.

The paper is organized as follows. In $\S 2$ we recall the required notation and facts about supersolvability of arrangements and reflection arrangements from OT92, $\S 4$, $\S 6]$. Further, in Proposition 2.5. we show that supersolvable arrangements behave well with respect to the product construction for arrangements. Using this fact, it is easy to construct non-supersolvable arrangements admitting modular elements of every possible rank, Remark 2.6.

Theorems 1.2 and 1.3 are proved in 93 in a sequence of lemmas. Here we provide a particularly useful inductive tool for showing that the reflection arrangement of a given reflection group $W$ is not supersolvable provided $W$ admits a suitable parabolic subgroup whose reflection arrangement is not supersolvable; cf. Lemma 3.1 .

For general information about arrangements and reflection groups we refer the reader to [Bou68] and OT92.

\section{ReCOlLECtions AND PRELIMINARIES}

2.1. Hyperplane arrangements. Let $V=\mathbb{C}^{\ell}$ be an $\ell$-dimensional complex vector space. A hyperplane arrangement is a pair $(\mathcal{A}, V)$, where $\mathcal{A}$ is a finite collection of hyperplanes in $V$. Usually, we simply write $\mathcal{A}$ in place of $(\mathcal{A}, V)$. We only consider central arrangements. The empty arrangement in $V$ is denoted by $\Phi_{\ell}$.

The lattice $L(\mathcal{A})$ of $\mathcal{A}$ is the set of subspaces of $V$ of the form $H_{1} \cap \cdots \cap H_{n}$, where $\left\{H_{1}, \ldots, H_{n}\right\}$ is a subset of $\mathcal{A}$. Note that $V$ belongs to $L(\mathcal{A})$ as the intersection of the empty collection of hyperplanes. The lattice $L(\mathcal{A})$ is a partially ordered set by reverse inclusion: $X \leq Y$ provided $Y \subseteq X$ for $X, Y \in L(\mathcal{A})$. We have a rank function on $L(\mathcal{A}): r(X):=\operatorname{codim}_{V}(X)$. The $\operatorname{rank} r(\mathcal{A})$ of $\mathcal{A}$ is the rank of a maximal element in $L(\mathcal{A})$ with respect to the partial order. With this definition $L(\mathcal{A})$ is a geometric lattice [OT92, p. 24]. The $\ell$-arrangement $\mathcal{A}$ is called essential provided $r(\mathcal{A})=\ell$.

The product $\mathcal{A}=\left(\mathcal{A}_{1} \times \mathcal{A}_{2}, V_{1} \oplus V_{2}\right)$ of two arrangements $\left(\mathcal{A}_{1}, V_{1}\right),\left(\mathcal{A}_{2}, V_{2}\right)$ is defined by

$$
\mathcal{A}:=\mathcal{A}_{1} \times \mathcal{A}_{2}=\left\{H_{1} \oplus V_{2} \mid H_{1} \in \mathcal{A}_{1}\right\} \cup\left\{V_{1} \oplus H_{2} \mid H_{2} \in \mathcal{A}_{2}\right\} ;
$$

see OT92, Def. 2.13]. Let $\mathcal{A}=\mathcal{A}_{1} \times \mathcal{A}_{2}$ be the product of the two arrangements $\mathcal{A}_{1}$ and $\mathcal{A}_{2}$. We define a partial order on $L\left(\mathcal{A}_{1}\right) \times L\left(\mathcal{A}_{2}\right)$ by $\left(X_{1}, X_{2}\right) \leq\left(Y_{1}, Y_{2}\right)$ provided $X_{1} \leq Y_{1}$ and $X_{2} \leq Y_{2}$. Then, by [T92, Prop. 2.14], there is a lattice isomorphism

$$
L\left(\mathcal{A}_{1}\right) \times L\left(\mathcal{A}_{2}\right) \cong L(\mathcal{A}) \quad \text { by } \quad\left(X_{1}, X_{2}\right) \mapsto X_{1} \oplus X_{2} .
$$


Note that $\mathcal{A} \times \Phi_{0}=\mathcal{A}$ for any arrangement $\mathcal{A}$. If $\mathcal{A}$ is of the form $\mathcal{A}=\mathcal{A}_{1} \times \mathcal{A}_{2}$, where $\mathcal{A}_{i} \neq \Phi_{0}$ for $i=1,2$, then $\mathcal{A}$ is called reducible; otherwise $\mathcal{A}$ is irreducible OT92, Def. 2.15]. For instance, the braid arrangement $\mathcal{A}\left(A_{\ell}\right)$ is the product of the empty 1-arrangement and an irreducible $(\ell-1)$-arrangement OT92, Ex. 2.16].

2.2. Reflection groups and reflection arrangements. The irreducible finite complex reflection groups were classified by Shephard and Todd [ST54]. Let $W \subseteq$ $\mathrm{GL}(V)$ be a finite complex reflection group. For $w \in W$, we write $\operatorname{Fix}(w):=\{v \in$ $V \mid w v=v\}$ for the fixed point subspace of $w$. For $U \subseteq V$ a subspace, we define the parabolic subgroup $W_{U}$ of $W$ by $W_{U}:=\{w \in W \mid U \subseteq \operatorname{Fix}(w)\}$.

The reflection arrangement $\mathcal{A}=\mathcal{A}(W)$ of $W$ in $V$ is the hyperplane arrangement consisting of the reflecting hyperplanes of the elements in $W$ acting as reflections on $V$. By Steinberg's Theorem [Ste60, Thm. 1.5], for $U \subseteq V$ a subspace, the parabolic subgroup $W_{U}$ is itself a complex reflection group, generated by the unitary reflections in $W$ that are contained in $W_{U}$. This allows us to identify the reflection arrangement $\mathcal{A}\left(W_{U}\right)$ of $W_{U}$ as a subarrangement of $\mathcal{A}$. This way, the lattice $L\left(\mathcal{A}\left(W_{U}\right)\right)$ of $\mathcal{A}\left(W_{U}\right)$ is identified with a sublattice of $L(\mathcal{A})$. We make these identifications throughout.

Note that for $X \in L(\mathcal{A})$, we have $\mathcal{A}\left(W_{X}\right)=\mathcal{A}_{X}:=\{H \in \mathcal{A} \mid X \subseteq H\}$; cf. OT92, Thm. 6.27, Cor. 6.28]. It follows that $L\left(\mathcal{A}\left(W_{X}\right)\right)=L\left(\mathcal{A}_{X}\right)=L(\mathcal{A})_{X}:=$ $\{Z \in L(\mathcal{A}) \mid X \subseteq Z\}$ by OT92, Lem. 2.11].

Following OT92, $\S 6.4$, App. C], we use the convention to label the $W$-orbit of $X \in L(\mathcal{A})$ by the type of the complex reflection group $W_{X}$. In that way we get a correspondence between the $W$-orbits in $L(\mathcal{A})$ and the conjugacy classes of parabolic subgroups of $W$.

2.3. Free and inductively free arrangements. Let $S=S\left(V^{*}\right)$ be the symmetric algebra of the dual space $V^{*}$ of $V$. If $x_{1}, \ldots, x_{\ell}$ is a basis of $V^{*}$, then we identify $S$ with the polynomial ring $\mathbb{C}\left[x_{1}, \ldots, x_{\ell}\right]$. Letting $S_{p}$ denote the $\mathbb{C}$-subspace of $S$ consisting of the homogeneous polynomials of degree $p$ (along with 0 ), we see that $S$ is naturally $\mathbb{Z}$-graded: $S=\bigoplus_{p \in \mathbb{Z}} S_{p}$, where $S_{p}=0$ for $p<0$.

Let $\operatorname{Der}(S)$ be the $S$-module of $\mathbb{C}$-derivations of $S$. For $i=1, \ldots, \ell$, let $D_{i}:=$ $\partial / \partial x_{i}$. Then $D_{1}, \ldots, D_{\ell}$ is a $\mathbb{C}$-basis of $\operatorname{Der}(S)$. We say that $\theta \in \operatorname{Der}(S)$ is homogeneous of polynomial degree $p$ provided $\theta=\sum_{i=1}^{\ell} f_{i} D_{i}$, where $f_{i} \in S_{p}$ for each $1 \leq i \leq \ell$. In this case we write $\operatorname{pdeg} \theta=p$. Let $\operatorname{Der}(S)_{p}$ be the $\mathbb{C}$-subspace of $\operatorname{Der}(S)$ consisting of all homogeneous derivations of polynomial degree $p$. Then $\operatorname{Der}(S)$ is a graded $S$-module: $\operatorname{Der}(S)=\bigoplus_{p \in \mathbb{Z}} \operatorname{Der}(S)_{p}$.

Following OT92, Def. 4.4], for $f \in S$, we define the $S$-submodule $D(f)$ of $\operatorname{Der}(S)$ by $D(f):=\{\theta \in \operatorname{Der}(S) \mid \theta(f) \in f S\}$. Let $\mathcal{A}$ be an arrangement in $V$. Then for $H \in \mathcal{A}$ we fix $\alpha_{H} \in V^{*}$ with $H=\operatorname{ker} \alpha_{H}$. The defining polynomial $Q(\mathcal{A})$ of $\mathcal{A}$ is given by $Q(\mathcal{A}):=\prod_{H \in \mathcal{A}} \alpha_{H} \in S$.

The module of $\mathcal{A}$-derivations of $\mathcal{A}$ is defined by $D(\mathcal{A}):=D(Q(\mathcal{A}))$. We say that $\mathcal{A}$ is free if the module of $\mathcal{A}$-derivations $D(\mathcal{A})$ is a free $S$-module. The notion of freeness was introduced by Saito in his seminal work $\underline{S 80}$.

With the $\mathbb{Z}$-grading of $\operatorname{Der}(S)$, the module of $\mathcal{A}$-derivations becomes a graded $S$ module $D(\mathcal{A})=\bigoplus_{p \in \mathbb{Z}} D(\mathcal{A})_{p}$, where $D(\mathcal{A})_{p}=D(\mathcal{A}) \cap \operatorname{Der}(S)_{p}$ [T92, Prop. 4.10]. If $\mathcal{A}$ is a free arrangement, then the $S$-module $D(\mathcal{A})$ admits a basis of $\ell$ homogeneous derivations, say $\theta_{1}, \ldots, \theta_{\ell}$ OT92, Prop. 4.18]. While the $\theta_{i}$ 's are not unique, their 
polynomial degrees pdeg $\theta_{i}$ are unique (up to ordering). This multiset is the set of exponents of the free arrangement $\mathcal{A}$ and is denoted by $\exp \mathcal{A}$.

There is a stronger notion of freeness, motivated by the so-called AdditionDeletion Theorem; see OT92, Thm. 4.51].

Definition 2.1. The class $\mathcal{I F}$ of inductively free arrangements is the smallest class of arrangements subject to

(i) $\Phi_{\ell} \in \mathcal{I F}$ for each $\ell \geq 0$;

(ii) if there exists an $H \in \mathcal{A}$ such that both the subarrangement $\mathcal{A} \backslash\{H\}$ of $\mathcal{A}$ and the restriction of $\mathcal{A}$ to $H, \mathcal{A}^{H}:=\left\{H^{\prime} \cap H \mid H^{\prime} \in \mathcal{A} \backslash\{H\}\right\}$, belong to $\mathcal{I F}$, and $\exp \mathcal{A}^{H} \subseteq \exp (\mathcal{A} \backslash\{H\})$, then $\mathcal{A}$ also belongs to $\mathcal{I} \mathcal{F}$.

Terao [Ter80, proved that each reflection arrangement is free. In HR12, we classified all inductively free reflection arrangements; cf. Theorem 1.1.

2.4. Supersolvable arrangements. Let $\mathcal{A}$ be an arrangement. Following OT92, $\S 2]$, we say that $X \in L(\mathcal{A})$ is modular provided $X+Y \in L(\mathcal{A})$ for every $Y \in L(\mathcal{A})$. (This is not the actual definition of a modular element, but it is equivalent to the definition in our case [OT92, Cor. 2.26].) Let $\mathcal{A}$ be a central (and essential) $\ell$-arrangement. The following notion is due to Stanley Sta72].

Definition 2.2. We say that $\mathcal{A}$ is supersolvable provided there is a maximal chain

$$
V=X_{0}<X_{1}<\ldots<X_{\ell-1}<X_{\ell}=\{0\}
$$

of modular elements $X_{i}$ in $L(\mathcal{A})$; cf. [OT92, Def. 2.32].

This terminology owes to the fact that the lattice of subgroups of a finite supersolvable group satisfies the condition in Definition 2.2

Remark 2.3. By [0T92, Ex. 2.28], $V,\{0\}$ and the members in $\mathcal{A}$ are always modular in $L(\mathcal{A})$. It follows that all 0 - 1-, and 2-arrangements are supersolvable.

As mentioned in the Introduction, supersolvable arrangements are always inductively free; see also OT92, Thm. 4.58]. In general, a free 3-arrangement need not be supersolvable as such an arrangement need not be inductively free; see OT92, Ex. 4.59].

The following observation is immediate from Definition 2.2 and Remark 2.3

Lemma 2.4. A 3-arrangement $\mathcal{A}$ is supersolvable if and only if there exists a modular rank 2 element in $L(\mathcal{A})$.

Thanks to [OT92, Prop. 4.28], free arrangements behave well with respect to the product construction for arrangements. This is also the case for supersolvable arrangements.

Proposition 2.5. Let $\mathcal{A}_{1}, \mathcal{A}_{2}$ be two arrangements. Then $\mathcal{A}=\mathcal{A}_{1} \times \mathcal{A}_{2}$ is supersolvable if and only if both $\mathcal{A}_{1}$ and $\mathcal{A}_{2}$ are supersolvable, and in that case the multiset of exponents of $\mathcal{A}$ is given by $\exp \mathcal{A}=\left\{\exp \mathcal{A}_{1}, \exp \mathcal{A}_{2}\right\}$.

Proof. Let $\mathcal{A}_{i}=\left(\mathcal{A}_{i}, V_{i}\right), \ell_{i}=\operatorname{dim} V_{i}$ for $i=1,2$, let $(\mathcal{A}, V)=\left(\mathcal{A}_{1} \times \mathcal{A}_{2}, V_{1} \oplus V_{2}\right)$, and let $\ell=\ell_{1}+\ell_{2}=\operatorname{dim} V$.

First suppose that both $\mathcal{A}_{1}$ and $\mathcal{A}_{2}$ are supersolvable with

$$
V_{i}=X_{i}^{0}<X_{i}^{1}<\ldots<X_{i}^{\ell_{i}}=\{0\}
$$


being a maximal chain of modular elements in $L\left(\mathcal{A}_{i}\right)$ for $i=1,2$. For $0 \leq j \leq \ell_{1}$ set $Z^{j}:=X_{1}^{j} \oplus V_{2}$ and for $\ell_{1}+1 \leq j \leq \ell$ set $Z^{j}:=\{0\} \oplus X_{2}^{j-\ell_{1}}=X_{2}^{j-\ell_{1}}$. By (2.1), $Z^{j} \in L(\mathcal{A})$ for each $0 \leq j \leq \ell$. We claim that

$$
V=Z^{0}<Z^{1}<\ldots<Z^{\ell}=\{0\}
$$

is a maximal chain of modular elements in $L(\mathcal{A})$. Clearly, this is a proper chain of elements in $L(\mathcal{A})$ of length $\ell$ by construction; cf. (2.1). Let $Y=Y_{1} \oplus Y_{2} \in L(\mathcal{A})$. Then, for $0 \leq j \leq \ell_{1}$,

$$
Z^{j}+Y=\left(X_{1}^{j} \oplus V_{2}\right)+\left(Y_{1} \oplus Y_{2}\right)=\left(X_{1}^{j}+Y_{1}\right) \oplus V_{2}
$$

belongs to $L(\mathcal{A})$ by (2.1), since $X_{1}^{j}$ is modular in $L\left(\mathcal{A}_{1}\right)$, so that $X_{1}^{j}+Y_{1} \in L\left(\mathcal{A}_{1}\right)$. Likewise,

$$
Z^{j}+Y=\left(\{0\} \oplus X_{2}^{j-\ell_{1}}\right)+\left(Y_{1} \oplus Y_{2}\right)=Y_{1} \oplus\left(X_{2}^{j-\ell_{1}}+Y_{2}\right)
$$

lies in $L(\mathcal{A})$, for $\ell_{1}+1 \leq j \leq \ell$ by (2.1). As $Y \in L(\mathcal{A})$ is arbitrary, $\mathcal{A}$ is supersolvable.

Now suppose that $\mathcal{A}$ is supersolvable. Let

$$
V=X^{0}<X^{1}<\ldots<X^{\ell-1}<X^{\ell}=\{0\}
$$

be a maximal chain of modular elements in $L(\mathcal{A})$. Then $X^{j}=X_{1}^{j} \oplus X_{2}^{j}$ for each $1 \leq j \leq \ell$ by (2.1). As each $X^{j}$ is modular in $L(\mathcal{A})$, for every $Y=Y_{1} \oplus Y_{2} \in L(\mathcal{A})$, the sum

$$
X^{j}+Y=\left(X_{1}^{j} \oplus X_{2}^{j}\right)+\left(Y_{1} \oplus Y_{2}\right)=\left(X_{1}^{j}+Y_{1}\right) \oplus\left(X_{2}^{j}+Y_{2}\right)
$$

belongs to $L(\mathcal{A})$. By (2.1), it follows that $X_{i}^{j}+Y_{i}$ belongs to $L\left(\mathcal{A}_{i}\right)$ for every $1 \leq j \leq \ell$ and $i=1,2$. Since $Y \in L(\mathcal{A})$ is arbitrary, each $X_{i}^{j}$ is modular in $L\left(\mathcal{A}_{i}\right)$ for every $1 \leq j \leq \ell$ and $i=1,2$. Since $\ell=\ell_{1}+\ell_{2}$, it follows from our construction that there are subsequences $1 \leq j_{1}<\ldots<j_{\ell_{1}}$ and $1 \leq k_{1}<\ldots<k_{\ell_{2}}$ of the integers from 1 to $\ell$ such that

$$
V_{1}=X_{1}^{0}<X_{1}^{j_{1}}<\ldots<X_{1}^{j_{\ell_{1}}}=\{0\}
$$

and

$$
V_{2}=X_{2}^{0}<X_{2}^{k_{1}}<\ldots<X_{2}^{k_{\ell_{2}}}=\{0\}
$$

are maximal chains of modular elements in $L\left(\mathcal{A}_{1}\right)$ and $L\left(\mathcal{A}_{2}\right)$, respectively. Thus both $\mathcal{A}_{1}$ and $\mathcal{A}_{2}$ are supersolvable, as desired.

Finally, the statement on the exponents follows from [OT92, Prop. 4.28] and the fact that supersolvable arrangements are free [JT84, Thm. 4.2].

Our final observation in this section shows that it is easy to construct an arrangement $\mathcal{A}$ which is not supersolvable but still admits modular elements of every possible rank.

Remark 2.6. Suppose that $\mathcal{A}_{i}=\left(\mathcal{A}_{i}, V_{i}\right)$ (for $\left.i=1,2\right)$ are arrangements so that $\mathcal{A}_{1}$ is supersolvable but $\mathcal{A}_{2}$ is not and that $\ell_{1}=\operatorname{dim} V_{1} \geq \ell_{2}=\operatorname{dim} V_{2}$. Consider the product $(\mathcal{A}, V)=\left(\mathcal{A}_{1} \times \mathcal{A}_{2}, V_{1} \oplus V_{2}\right)$. While $\mathcal{A}$ is again not supersolvable, thanks to Proposition [2.5, it is easy to see that $L(\mathcal{A})$ admits modular elements of every possible rank $r$ for any $0 \leq r \leq \ell_{1}+\ell_{2}=r(\mathcal{A})$, for let

$$
V_{1}=X_{0}<X_{1}<\ldots<X_{\ell_{1}}=\{0\}
$$

be a maximal chain of modular elements in $L\left(\mathcal{A}_{1}\right)$. Then, by (2.1), $Z_{s}:=X_{s} \oplus V_{2}$ and $Z_{t}^{\prime}:=X_{t} \oplus\{0\}$ belong to $L(\mathcal{A})$ for each $0 \leq s \leq \ell_{2} \leq \ell_{1}$ and $1 \leq t \leq \ell_{1}$. Since $\operatorname{codim}_{V} Z_{s}=\operatorname{codim}_{V_{1}} X_{s}=s$ and $\operatorname{codim}_{V} Z_{t}^{\prime}=\operatorname{codim}_{V_{1}} X_{t}+\operatorname{dim} V_{2}=t+\ell_{2}$, the 
rank of $Z_{s}$ is $s$ for each $0 \leq s \leq \ell_{2}$, and that of $Z_{t}^{\prime}$ is $\ell_{2}+t$ for each $1 \leq t \leq \ell_{1}$. Now let $Y=Y_{1} \oplus Y_{2} \in L(\mathcal{A})$ be arbitrary. Then, since each $X_{s}$ is modular in $L\left(\mathcal{A}_{1}\right)$, it follows from (2.1) that

$$
Z_{s}+Y=\left(X_{s} \oplus V_{2}\right)+\left(Y_{1} \oplus Y_{2}\right)=\left(X_{s}+Y_{1}\right) \oplus V_{2} \in L(\mathcal{A})
$$

and

$$
Z_{t}^{\prime}+Y=\left(X_{t} \oplus\{0\}\right)+\left(Y_{1} \oplus Y_{2}\right)=\left(X_{t}+Y_{1}\right) \oplus Y_{2} \in L(\mathcal{A}) .
$$

Hence $Z_{s}$ is modular of rank $s$ for $0 \leq s \leq \ell_{2}$ and $Z_{t}^{\prime}$ is modular of rank $\ell_{2}+t$ for $1 \leq t \leq \ell_{1}$. In particular, $L(\mathcal{A})$ admits modular elements of every possible rank.

\section{Proofs of Theorems 1.2 and 1.3}

Our first result allows us to conclude that $\mathcal{A}(W)$ is not supersolvable given that the reflection arrangement of a suitable parabolic subgroup of $W$ is not supersolvable. While this is an elementary observation, it is nevertheless a rather effective inductive tool.

Lemma 3.1. Let $W$ be a complex reflection group and $\mathcal{A}=\mathcal{A}(W)$ its reflection arrangement. Suppose that there are $X \in L(\mathcal{A})$ and $r \in \mathbb{N}$ such that $L\left(\mathcal{A}\left(W_{X}\right)\right)$ does not admit modular elements of rank $r$ and that every $W$-orbit of elements in $L(\mathcal{A})$ of rank $r$ meets $L\left(\mathcal{A}\left(W_{X}\right)\right)$. Then $L(\mathcal{A})$ does not admit modular elements of rank $r$. In particular, $\mathcal{A}$ is not supersolvable.

Proof. Let $Y \in L(\mathcal{A})$ be of rank $r$. Recall from Section 2.2 that $L\left(\mathcal{A}\left(W_{X}\right)\right)=$ $L(\mathcal{A})_{X}$. Therefore, by our hypotheses there exists a $w \in W$ so that $w Y \in L(\mathcal{A})_{X}$, and there is a $Z \in L(\mathcal{A})_{X}$ so that $U:=w Y+Z \notin L(\mathcal{A})_{X}$. It suffices to show that $U \notin L(\mathcal{A})$, as then $Y+w^{-1} Z$ does not belong to $L(\mathcal{A})$, and so $Y$ is not modular. So, for a contradiction, suppose that $U \in L(\mathcal{A})$. Since both $w Y$ and $Z$ belong to $L(\mathcal{A})_{X}$, we have $X \subseteq w Y+Z=U$, and so $U \in L(\mathcal{A})_{X}$ which is absurd, since $U \notin L(\mathcal{A})_{X}$ by construction. Consequently, $U \notin L(\mathcal{A})$, as desired.

Note that the first condition on $L\left(\mathcal{A}\left(W_{X}\right)\right)$ in Lemma 3.1 says that $\mathcal{A}\left(W_{X}\right)$ is not supersolvable.

Lemma 3.2. If $W$ is of type $D_{4}, F_{4}, H_{3}$, or $W=G_{25}$ or $G_{26}$, then there are no modular elements in $L(\mathcal{A}(W))$ of rank 2 . In particular, $\mathcal{A}(W)$ is not supersolvable.

Proof. Let $\mathcal{A}=\mathcal{A}(W)$. Using the explicit data on the $W$-orbits in $L(\mathcal{A})$ from OT92, $\S 6$, App. C], we choose an orbit representative $X$ for each $W$-orbit in $L(\mathcal{A})$ of elements of rank 2 , and give an explicit $Y \in L(\mathcal{A})$ in each case so that $X+Y \notin L(\mathcal{A})$. We denote the coordinate functions in $S$ simply by $a, b, c$, etc., and for $f \in V^{*}$, we write $H_{f}$ for $\operatorname{ker} f$.

(i) Let $W$ be of type $D_{4}$. Jambu and Terao have already observed that $\mathcal{A}=$ $\mathcal{A}\left(D_{4}\right)$ is not supersolvable [JT84, Ex. 5.5]. We give a different, elementary argument showing that $L(\mathcal{A})$ does not admit modular elements of rank 2 . The defining polynomial of $\mathcal{A}=\mathcal{A}\left(D_{4}\right)$ is

$$
\begin{gathered}
Q\left(D_{4}\right):=(a-b)(a+b)(a-c)(a+c)(a-d)(a+d) \\
(b-c)(b+c)(b-d)(b+d)(c-d)(c+d) .
\end{gathered}
$$

There are four $W$-orbits of elements of rank 2 in $L(\mathcal{A})$, corresponding to three conjugacy classes of parabolic subgroups of $W$ of type $A_{1}^{2}$ and one of type $A_{2}$. The conjugacy classes of type $A_{1}^{2}$ are fused by the action of the group of graph 
automorphisms of $W$. It thus suffices to only consider one orbit in $L(\mathcal{A})$ with a parabolic subgroup of type $A_{1}^{2}$ and another one with point stabilizer of type $A_{2}$, where representatives of these orbits are given by

$$
X_{1}=H_{a+b} \cap H_{a-b} \quad \text { and } \quad X_{2}=H_{a-b} \cap H_{b-c},
$$

respectively. One readily checks that $X_{1}+\left(H_{b+d} \cap H_{b-d}\right)=H_{b} \notin \mathcal{A}$, as well as that $X_{2}+\left(H_{a+b} \cap H_{c-d} \cap H_{c+d}\right)=H_{a+b-2 c} \notin \mathcal{A}$. Thus both $X_{1}$ and $X_{2}$ are not modular.

(ii) Let $W$ be of type $F_{4}$. The defining polynomial of $\mathcal{A}=\mathcal{A}\left(F_{4}\right)$ is given by

$$
\begin{aligned}
Q\left(F_{4}\right) & :=a b c d(a+b)(b+c)(c+d)(b+2 c)(a+b+c)(b+c+d)(a+b+2 c) \\
& (a+b+c+d)(b+2 c+d)(a+2 b+2 c)(a+b+2 c+d)(b+2 c+2 d) \\
& (a+2 b+2 c+d)(a+b+2 c+2 d)(a+2 b+3 c+d)(a+2 b+2 c+2 d) \\
& (a+2 b+3 c+2 d)(a+2 b+4 c+2 d)(a+3 b+4 c+2 d)(2 a+3 b+4 c+2 d) .
\end{aligned}
$$

There are four $W$-orbits of elements of rank 2 in $L(\mathcal{A})$ (cf. [OT92, Table C.9]) corresponding to the four conjugacy classes of parabolic subgroups of $W$ of type $A_{2}, \widetilde{A}_{2}, A_{1} \times \widetilde{A}_{1}$, and $B_{2}$ with representatives

$X_{1}=H_{a} \cap H_{b}, \quad X_{2}=H_{c} \cap H_{d}, \quad X_{3}=H_{c+d} \cap H_{a+2 b+2 c+2 d}$, and $X_{4}=H_{a} \cap H_{b+c}$, respectively. We claim that none of these elements is modular. First note that we have $X_{1}+X_{3}=H_{a+2 b} \notin \mathcal{A}$, so both $X_{1}$ and $X_{3}$ are not modular. Moreover, one checks that $X_{2}+\left(H_{a+2 b+3 c+d} \cap H_{a+2 b+2 c+2 d}\right)=H_{c-d} \notin \mathcal{A}$ and also that $X_{4}+\left(H_{b} \cap H_{a+b+c+d} \cap H_{a+2 b+4 c+2 d}\right)=H_{a-2 b-2 c} \notin \mathcal{A}$, and so $X_{2}$ and $X_{4}$ are not modular either.

(iii) Let $W$ be of type $H_{3}$. The defining polynomial of $\mathcal{A}=\mathcal{A}\left(H_{3}\right)$ is

$$
\begin{aligned}
Q\left(H_{3}\right) & :=a b c(a-\omega b)(a-(\omega+1) b)(b+c)(a+b)(a-\omega b-\omega c) \\
& (a-(\omega+1) b-(\omega+1) c)(a+b+c)(a-\omega b-(\omega+1) c)(a-\omega b+c) \\
& (a+b+(\omega+2) c)(a+b-(\omega+1) c)(a-2(\omega+1) b-(\omega+1) c),
\end{aligned}
$$

where $\omega=\eta^{2}+\eta^{3}$ and $\eta$ is a primitive 5 -th root of unity. There are three $W$ orbits of elements of rank 2 in $L(\mathcal{A})$; cf. OT92, Table C.4]. They correspond to the three conjugacy classes of parabolic subgroups of $W$ of types $I_{2}(5), A_{2}$ and $A_{1}^{2}$ with respective representatives

$$
X_{1}=H_{a} \cap H_{b}, \quad X_{2}=H_{a} \cap H_{a-\omega b-(\omega+1) c}, \text { and } X_{3}=H_{c} \cap H_{a-2(\omega+1) b-(\omega+1) c} .
$$

One calculates that $X_{1}+X_{3}=H_{a-2(\omega+1) b}$ is not in $\mathcal{A}$ and moreover that $X_{2}+$ $\left(H_{a+b} \cap H_{a-\omega b+c}\right)=H_{2 a-(\omega-1) b+c} \notin \mathcal{A}$; hence none of these elements is modular.

(iv) Let $W=G_{25}$. The defining polynomial of $\mathcal{A}=\mathcal{A}\left(G_{25}\right)$ is

$$
\begin{aligned}
Q\left(G_{25}\right) & :=a b c(a+b+c)(a+b+\zeta c)\left(a+b+\zeta^{2} c\right)(a+\zeta b+c) \\
& (a+\zeta b+\zeta c)\left(a+\zeta b+\zeta^{2} c\right)\left(a+\zeta^{2} b+c\right)\left(a+\zeta^{2} b+\zeta c\right)\left(a+\zeta^{2} b+\zeta^{2} c\right),
\end{aligned}
$$

where $\zeta$ is a primitive 3 -rd root of unity. There are two $W$-orbits of elements of rank 2 in $L(\mathcal{A})$ OT92, Table C.6]. They correspond to the two conjugacy classes of parabolic subgroups of $W$ isomorphic to $C(3)^{2}$ and $G_{4}$ with respective representatives

$$
X_{1}=H_{a} \cap H_{b} \quad \text { and } \quad X_{2}=H_{c} \cap H_{a+b+c} .
$$

Since $X_{1}+X_{2}=H_{a+b} \notin \mathcal{A}$, both $X_{1}$ and $X_{2}$ are not modular. 
(v) Let $W=G_{26}$. Falk and Randell [FR87, (3.3)] already asserted that $\mathcal{A}=$ $\mathcal{A}\left(G_{26}\right)$ is not supersolvable. We give a different, elementary argument showing that $L(\mathcal{A})$ does not admit modular elements of rank 2. The defining polynomial of $\mathcal{A}$ is

$$
\begin{aligned}
Q\left(G_{26}\right) & :=a b c(a-b)(a-c)(b-c)(a-\zeta b)\left(a-\zeta^{2} b\right)(a-\zeta c)\left(a-\zeta^{2} c\right)(b-\zeta c) \\
& \left(b-\zeta^{2} c\right)(a+b+c)(a+b+\zeta c)\left(a+b+\zeta^{2} c\right)(a+\zeta b+c)(a+\zeta b+\zeta c) \\
& \left(a+\zeta b+\zeta^{2} c\right)\left(a+\zeta^{2} b+c\right)\left(a+\zeta^{2} b+\zeta c\right)\left(a+\zeta^{2} b+\zeta^{2} c\right),
\end{aligned}
$$

where $\zeta$ is a primitive 3 -rd root of unity. There are three $W$-orbits of elements of rank 2 in $L(\mathcal{A})$ OT92, Table C.7]. They correspond to the three conjugacy classes of parabolic subgroups of $W$ isomorphic to $A_{1} \times C(3), G_{4}$, and $G(3,1,2)$ with representatives

$$
X_{1}=H_{b} \cap H_{a-\zeta c}, \quad X_{2}=H_{c} \cap H_{a+\zeta b+c}, \quad \text { and } \quad X_{3}=H_{a} \cap H_{b},
$$

respectively. One checks that $X_{2}+X_{3}=H_{a+\zeta b} \notin \mathcal{A}$ and moreover that $X_{1}+$ $\left(H_{a-b} \cap H_{b-\zeta^{2} c}\right)=H_{a-(\zeta+2) b-\zeta c} \notin \mathcal{A}$, and so none of these elements is modular.

This completes the proof of Lemma 3.2 .

Armed with Lemmas 3.1 and 3.2 we can now readily derive that a large class of unitary reflection groups does not admit a supersolvable reflection arrangement. For $W$ of types $E_{6}, E_{7}, E_{8}$, and $H_{4}$, Falk and Randell had raised this question in [FR87, (2.1)].

Lemma 3.3. If $W$ is of type $D_{\ell}$, for $\ell \geq 5, E_{6}, E_{7}, E_{8}, H_{4}$, or $W=G_{32}$, then there are no modular elements in $L(\mathcal{A}(W))$ of rank 2. In particular, $\mathcal{A}(W)$ is not supersolvable.

Proof. Let $\mathcal{A}=\mathcal{A}(W)$. If $W$ is one of the Weyl groups as in the statement, let $W_{X}$ be a parabolic subgroup of $W$ of type $D_{4}$, if $W=H_{4}$, let $W_{X}=H_{3}$, and if $W=G_{32}$, then let $W_{X}=G_{25}$. By Lemma 3.2. $L\left(\mathcal{A}\left(W_{X}\right)\right)$ does not admit modular elements of rank 2. From the information on the $W$-orbits in $L(\mathcal{A})$ given in OT92, $\S 6$, App. C], we infer that with these choices, every $W$-orbit of elements in $L(\mathcal{A})$ of rank 2 meets the sublattice $L\left(\mathcal{A}\left(W_{X}\right)\right)$. The desired result follows from Lemma 3.1

Proof of Theorem 1.2. Thanks to Proposition 2.5 the question of supersolvability reduces to the case when $\mathcal{A}=\mathcal{A}(W)$ is irreducible. Therefore, we may assume that $W$ is irreducible.

(i) Let $W$ be of rank at most 2. Then $\mathcal{A}(W)$ is supersolvable by Remark 2.3 ,

(ii) Let $W$ be an irreducible Coxeter group of rank at least 3. Fadell and Neuwirth [FN62 proved that the braid arrangement $\mathcal{A}\left(A_{\ell}\right)$ is fiber-type, and Brieskorn [Br73] showed that the reflection arrangement of the hyperoctahedral group $\mathcal{A}\left(B_{\ell}\right)$ is also fiber-type. Thus $\mathcal{A}\left(A_{\ell}\right)$ and $\mathcal{A}\left(B_{\ell}\right)$ are supersolvable by Ter86]. It follows from Lemmas 3.2 and 3.3 that for all other types $\mathcal{A}(W)$ is not supersolvable.

(iii) Let $W$ be a monomial group $G(r, p, \ell)$. Note that the reflection arrangements for $G(r, 1, \ell)$ and $G(r, p, \ell)$ for $p \neq r$ coincide. It thus suffices to consider the case $G(r, 1, \ell)$. Let $\mathcal{A}=\mathcal{A}(G(r, 1, \ell))$ for $r, \ell \geq 3$. Orlik and Solomon OS83, §4] showed that $\mathcal{A}$ is fiber-type, and so $\mathcal{A}$ is supersolvable. We give an elementary, 
direct argument for the supersolvability of $\mathcal{A}$. Let $H_{i}=\operatorname{ker} x_{i}$ and define $X_{i}=$ $H_{1} \cap H_{2} \cap \ldots \cap H_{i}$ for $1 \leq i \leq \ell$. Set $X_{0}=V$. We claim that

$$
V=X_{0}<X_{1}<\ldots<X_{\ell}=\{0\}
$$

is a maximal chain of modular elements in $L(\mathcal{A})$. Let $\zeta=e^{2 \pi i / r}$ be a primitive $r$-th root of unity. The hyperplanes of $\mathcal{A}$ are of the form $H_{i}=\operatorname{ker} x_{i}$ for $1 \leq i \leq \ell$ and $H_{i, j}(m)=\operatorname{ker}\left(x_{i}-\zeta^{m} x_{j}\right)$ for $1 \leq i<j \leq \ell$ and $0 \leq m \leq r-1$. So for an intersection of such hyperplanes $Y \in L(\mathcal{A})$, the coordinates of the subspace $Y$ are either 0 or some coincide, while others differ by a power of $\zeta$. Now $X_{k}$ consists of all vectors in $V$ whose first $k$ coordinates are zero and all others are arbitrary, for $1 \leq k \leq \ell$. Thus the relations among the coordinates of a vector in the sum $X_{k}+Y$ of the two subspaces are the same as for the ones in $Y$ for the first $k$ coordinates, and for higher indices there are no restrictions. So $X_{k}+Y$ coincides with the intersection of those hyperplanes $H_{i}$ and $H_{i, j}(m)$ that define $Y$ subject to the requirement that all occurring indices $i, j$ are at most $k$. In particular, $X_{k}+Y$ belongs again to $L(\mathcal{A})$, and so $X_{k}$ is modular for $1 \leq k \leq \ell$, as claimed.

Note that this argument also applies to the braid arrangement $\mathcal{A}(G(1,1, \ell)$ ) (cf. OT92, Ex. 2.33]), as well as to the reflection arrangement of the hyperoctahedral group $\mathcal{A}(G(2,1, \ell))$.

By Theorem 1.1 and JT84, Thm. 4.2], the reflection arrangements of the monomial groups $G(r, r, \ell)$ for $r, \ell \geq 3$ are not supersolvable.

(iv) Finally, let $W$ be an irreducible, non-real, unitary reflection group of rank at least 3. By Theorem 1.1 and [JT84], the reflection arrangements of $G_{24}, G_{27}$, $G_{29}, G_{31}, G_{33}$, and $G_{34}$ are not supersolvable; neither are the ones of $G_{25}, G_{26}$ and $G_{32}$ by Lemmas 3.2 and 3.3 .

This completes the proof of Theorem 1.2 .

We now attend to Theorem 1.3. Our first observation is immediate from Theorem 1.2 and Lemma 2.4

Lemma 3.4. If $W$ is a monomial group $G(r, r, 3)$ for $r \geq 3, G_{24}$ or $G_{27}$, then there are no modular elements in $L(\mathcal{A}(W))$ of rank 2 .

Lemma 3.5. If $W$ is a monomial group $G(r, r, 4)$ for $r \geq 3, G_{29}$ or $G_{31}$, then there are no modular elements in $L(\mathcal{A}(W))$ of rank 2 .

Proof. Let $\mathcal{A}=\mathcal{A}(W)$. As before, we denote the coordinate functions in $S$ simply by $a, b, c$, and $d$, and for $f \in V^{*}$, we write $H_{f}$ for $\operatorname{ker} f$.

(i) Let $W=G(r, r, 4)$ for $r \geq 3$. There are three $W$-orbits in $L(\mathcal{A})$ of elements of rank 2 corresponding to the conjugacy classes of parabolic subgroups of types $A_{1}^{2}, A_{2}$ and $I_{2}(r)$; cf. OT92, $\left.\$ 6.4\right]$. Let $W_{X}=G(r, r, 3)$ be a parabolic subgroup, where $X \in L(\mathcal{A})$ is of rank 3 . There are two $W_{X}$-orbits in $L\left(\mathcal{A}\left(W_{X}\right)\right)$ of elements of rank 2 corresponding to the conjugacy classes of parabolic subgroups of types $A_{2}$ and $I_{2}(r)$. It follows from Lemma 3.4 and the argument in the proof of Lemma 3.1 that for each $Y \in L(\mathcal{A})$ of rank 2 whose $W$-orbit meets $L\left(\mathcal{A}\left(W_{X}\right)\right), Y$ is not modular. This leaves the $W$-orbit in $L(\mathcal{A})$ whose elements have a point stabilizer of type $A_{1}^{2}$ to be considered. One such element is given by $Y=H_{a-b} \cap H_{c-d}$. But $Y+\left(H_{b-c} \cap H_{a-d}\right)=H_{a-b+c-d} \notin \mathcal{A}$, so $Y$ is not modular.

(ii) Let $W=G_{29}$. There are three $W$-orbits in $L(\mathcal{A})$ of elements of rank 2 corresponding to the conjugacy classes of parabolic subgroups of types $A_{1}^{2}, A_{2}$ and $B_{2}$; cf. [T92, Table C.10]. Let $W_{X}=G(4,4,3)$ be a parabolic subgroup of $W$, 
where $X$ has rank 3. There are two $W_{X}$-orbits in $L\left(\mathcal{A}\left(W_{X}\right)\right)$ of elements of rank 2 corresponding to the conjugacy classes of parabolic subgroups of types $A_{2}$ and $B_{2}$. It follows from Lemma 3.4 and the argument in the proof of Lemma 3.1 that for each $Y \in L(\mathcal{A})$ of rank 2 whose $W$-orbit meets $L\left(\mathcal{A}\left(W_{X}\right)\right), Y$ is not modular. This leaves the $W$-orbit whose point stabilizer is of type $A_{1}^{2}$ to be considered. Let $i=\sqrt{-1}$. The defining polynomial of $\mathcal{A}$ is given by

$$
\begin{aligned}
Q\left(G_{29}\right) & :=a b c d(a-b)(a-c)(a-d)(b-c)(b-d)(c-d) \\
& (a+c)(a+b)(a+d)(b+c)(b+d)(c+d) \\
& (a-b+i c+i d)(a-b+i c-i d)(a-b-i c-i d)(a-b-i c+i d) \\
& (a+b+i c+i d)(a+b-i c-i d)(a+b-i c+i d)(a+b+i c-i d) \\
& (a-i b+i c+d)(a-i b-c-i d)(a-i b-c+i d)(a-i b+i c-d) \\
& (a-i b-i c+d)(a-i b+c-i d)(a-i b-i c-d)(a+i b-c+i d) \\
& (a+i b-c-i d)(a+i b-i c+d)(a+i b-i c-d)(a+i b+c+i d) \\
& (a+i b+i c+d)(a+i b+i c-d)(a-i b+c+i d)(a+i b+c-i d) .
\end{aligned}
$$

A member in $L(\mathcal{A})$ with point stabilizer $A_{1}^{2}$ is given by $Y=H_{a-b+i c+i d} \cap H_{a+i b-c-i d}$. One checks that $Y+\left(H_{a+i b-i c+d} \cap H_{b-d}\right)=H_{a+(2 i-1) b-i c-(i-2) d} \notin \mathcal{A}$, so $Y$ is not modular.

(iii) Let $W=G_{31}$. Let $i=\sqrt{-1}$. The defining polynomial of $\mathcal{A}=\mathcal{A}\left(G_{31}\right)$ is given by

$$
\begin{aligned}
Q\left(G_{31}\right) & :=a b c d(a-b)(a-c)(a-d)(b-c)(b-d)(c-d) \\
& (a+b)(a+c)(a+d)(b+c)(b+d)(c+d) \\
& (a-i b)(a-i c)(a-i d)(b-i c)(b-i d)(c-i d) \\
& (a+i b)(a+i c)(a+i d)(b+i c)(b+i d)(c+i d) \\
& (a-b-c-d)(a-b+c+d)(a-b+c-d)(a-b-c+d) \\
& (a+b+c+d)(a+b-c+d)(a+b-c-d)(a+b+c-d) \\
& (a-b-i c-i d)(a-b+i c+i d)(a-b-i c+i d)(a-b+i c-i d) \\
& (a+b-i c-i d)(a+b+i c+i d)(a+b+i c-i d)(a+b-i c+i d) \\
& (a-i b-c-i d)(a-i b+c-i d)(a-i b-c+i d)(a-i b+c+i d) \\
& (a-i b+i c+d)(a-i b+i c-d)(a-i b-i c+d)(a-i b-i c-d) \\
& (a+i b+c-i d)(a+i b-c-i d)(a+i b-c+i d)(a+i b+c+i d) \\
& (a+i b-i c+d)(a+i b-i c-d)(a+i b+i c-d)(a+i b+i c+d) .
\end{aligned}
$$

There are three $W$-orbits of elements of rank 2 in $L(\mathcal{A})$; cf. OT92, Table C.12]. They correspond to the three conjugacy classes of parabolic subgroups of $W$ of types $A_{1}^{2}, A_{2}$, and $G(4,2,2)$ with respective representatives

$$
X_{1}=H_{a} \cap H_{b-c}, \quad X_{2}=H_{a+i d} \cap H_{a+b-c-d}, \quad \text { and } \quad X_{3}=H_{a} \cap H_{a+i b} .
$$

One checks that $X_{1}+X_{2}=H_{2 a+(1+i) b-(1+i) c} \notin \mathcal{A}$, so that $X_{1}$ and $X_{2}$ are not modular. Further, one calculates that $X_{3}+\left(H_{a-b-c-d} \cap H_{a-i c} \cap H_{a-b-c+d}\right)=$ $H_{2 a+(i-1) b} \notin \mathcal{A}$, so $X_{3}$ is not modular either.

This completes the proof of Lemma 3.5 . 
Lemma 3.6. If $W$ is a monomial group $G(r, r, \ell)$ for $r \geq 3, \ell \geq 5, G_{33}$, or $G_{34}$, then there are no modular elements in $L(\mathcal{A}(W))$ of rank 2 .

Proof. Let $\mathcal{A}=\mathcal{A}(W)$. If $W=G(r, r, \ell)$ for $r \geq 3, \ell \geq 5, G_{33}$, and $G_{34}$, let $W_{X}$ be the parabolic subgroup $W_{X}=G(r, r, 4), W_{X}=W\left(D_{4}\right)$, and $W_{X}=W\left(D_{4}\right)$, respectively. By Lemmas 3.2 and 3.5, $L\left(\mathcal{A}\left(W_{X}\right)\right)$ does not admit modular elements of rank 2. From the information on the $W$-orbits in $L(\mathcal{A})$ given in OT92, $\S 6$, Table C.14, C.17], we infer that with these choices, every $W$-orbit of elements in $L(\mathcal{A})$ of rank 2 meets the sublattice $L\left(\mathcal{A}\left(W_{X}\right)\right)$. The desired result follows from Lemma 3.1 .

Proof of Theorem 1.3. The forward implication is clear from Definition 2.2.

The reverse implication follows for general central arrangements of rank up to 3 by Remark 2.3 and Lemma 2.4. So assume that $\mathcal{A}(W)$ is irreducible of rank at least 4. It follows readily from Theorem 1.2 and Lemmas 3.2 through 3.6 that if $\mathcal{A}$ is not supersolvable, then there are no modular elements of rank 2 in $L(\mathcal{A})$.

While Theorem 1.3 asserts that an irreducible reflection arrangement is supersolvable precisely when its lattice admits a modular element of rank 2 , this assertion is false for reducible reflection arrangements; see Remark 2.6.

The defining polynomials of the reflection arrangements for $F_{4}, H_{3}, G_{25}, G_{26}$ and $G_{31}$ used in the proofs of Lemmas 3.2 and 3.5 were obtained using the functionality for complex reflection groups provided by the CHEVIE package in GAP (and some GAP code by J. Michel) (see $\mathrm{S}^{+} 97$ and $\left[\mathrm{GHL}^{+} 96\right.$ ).

\section{REFERENCES}

[Be06] D. Bessis, Finite complex reflection arrangements are $K(\pi, 1)$, Annals of Math., to appear.

[Bou68] N. Bourbaki, Éléments de mathématique. Fasc. XXXIV. Groupes et algèbres de Lie. Chapitre IV: Groupes de Coxeter et systèmes de Tits. Chapitre V: Groupes engendrés par des réflexions. Chapitre VI: systèmes de racines (French), Actualités Scientifiques et Industrielles, No. 1337, Hermann, Paris, 1968. MR.0240238 (39 \#1590)

[Br73] Egbert Brieskorn, Sur les groupes de tresses [d'après V. I. Arnol'd] (French), Séminaire Bourbaki, 24ème année (1971/1972), Exp. No. 401, Lecture Notes in Math., Vol. 317, Springer, Berlin, 1973, pp. 21-44. MR0422674 (54 \#10660)

[Del72] Pierre Deligne, Les immeubles des groupes de tresses généralisés (French), Invent. Math. 17 (1972), 273-302. MR.0422673 (54 \#10659)

[FN62] Edward Fadell and Lee Neuwirth, Configuration spaces, Math. Scand. 10 (1962), 111118. MR0141126 (25 \#4537)

[FR85] Michael Falk and Richard Randell, The lower central series of a fiber-type arrangement, Invent. Math. 82 (1985), no. 1, 77-88, DOI 10.1007/BF01394780. MR808110 (87c:32015b)

[FR87] Michael Falk and Richard Randell, On the homotopy theory of arrangements. Complex analytic singularities, 101-124, Adv. Stud. Pure Math., 8, North-Holland, Amsterdam, 1987. MR 0894288

[GHL+96] Meinolf Geck, Gerhard Hiss, Frank Lübeck, Gunter Malle, and Götz Pfeiffer, CHEVIE - a system for computing and processing generic character tables, Computational methods in Lie theory (Essen, 1994). Appl. Algebra Engrg. Comm. Comput. 7 (1996), no. 3, 175-210, DOI 10.1007/BF01190329. MR.1486215 (99m:20017)

[HR12] T. Hoge and G. Röhrle, On inductively free reflection arrangements, J. Reine Angew. Math., DOI 10.1515/ crelle-2013-0022, to appear.

[JT84] Michel Jambu and Hiroaki Terao, Free arrangements of hyperplanes and supersolvable lattices, Adv. in Math. 52 (1984), no. 3, 248-258, DOI 10.1016/0001-8708(84)90024-0. MR:744859(86c:32004) 
[OS83] Peter Orlik and Louis Solomon, Coxeter arrangements, Singularities, Part 2 (Arcata, Calif., 1981), Proc. Sympos. Pure Math., vol. 40, Amer. Math. Soc., Providence, RI, 1983, pp. 269-291. MR713255 (85b:32016)

[OT92] Peter Orlik and Hiroaki Terao, Arrangements of hyperplanes, Grundlehren der Mathematischen Wissenschaften [Fundamental Principles of Mathematical Sciences], vol. 300, Springer-Verlag, Berlin, 1992. MR1217488 (94e:52014)

[S80] Kyoji Saito, Theory of logarithmic differential forms and logarithmic vector fields, J. Fac. Sci. Univ. Tokyo Sect. IA Math. 27 (1980), no. 2, 265-291. MR.586450 (83h:32023)

[S+97] M. Schönert et al., GAP - Groups, Algorithms, and Programming - version 3 release 4, 1997.

[ST54] G. C. Shephard and J. A. Todd, Finite unitary reflection groups, Canadian J. Math. 6 (1954), 274-304. MR0059914 (15,600b)

[Sta72] R. P. Stanley, Supersolvable lattices, Algebra Universalis 2 (1972), 197-217. MR0309815 (46 \#8920)

[Ste60] Robert Steinberg, Invariants of finite reflection groups, Canad. J. Math. 12 (1960), 616-618. MR0117285(22 \#8066)

[Ter80] Hiroaki Terao, Arrangements of hyperplanes and their freeness. I, J. Fac. Sci. Univ. Tokyo Sect. IA Math. 27 (1980), no. 2, 293-312. MR586451 (84i:32016a)

[Ter86] Hiroaki Terao, Modular elements of lattices and topological fibration, Adv. in Math. 62 (1986), no. 2, 135-154, DOI 10.1016/0001-8708(86)90097-6. MR865835 (88b:32032)

Fakultät für Mathematik, Ruhr-Universität Bochum, D-44780 Bochum, Germany

Current address: Institut für Algebra, Zahlentheorie und Diskrete Mathematik, Fakultät für Mathematik und Physik, Leibniz Universität Hannover, Welfengarten 1, 30167 Hannover, Germany

E-mail address: hoge@math.uni-hannover.de

FAkultät Für Mathematik, Ruhr-Universität Bochum, D-44780 Bochum, Germany

E-mail address: gerhard.roehrle@rub.de 